\title{
Hyperglycaemic Hemichorea
}

\author{
Joana Silva Marques, Nuno Monteiro, Ana Nunes, João Machado, João Olivério, Ana Sofia Martins, António Correia \\ Internal Medicine Department of Tondela-Viseu Hospital Centre, Viseu, Portugal
}

\section{Received: 07/12/2017}

Accepted: $18 / 12 / 2017$

Published: 06/02/2018

How to cite this article: Marques J, Monteiro N, Nunes A, Machado J, Olivéiro J, Martins AS, Correia A. Hyperglicaemic hemichorea. EJCRIM 2018;5: doi:10.12890/2018_000807.

Conflicts of Interests: The Authors declare that there are no competing interests.

This article is licensed under a Commons Attribution Non-Commercial 4.0 License

\section{ABSTRACT}

Background: Hyperkinetic disorders such as hemichorea can be caused by cerebrovascular, infectious or inflammatory diseases or by metabolic conditions such as hyperglycaemia. Hyperglycaemic hemichorea is a rare movement disorder which is frequently misdiagnosed. It is characterized by involuntary, continuous, non-patterned movements on one side of the body, basal ganglia lesions seen on head CT or MRI, and clinical improvement after blood glucose normalization. We describe the case of a female patient with uncontrolled diabetes who presented with hemichorea.

Case presentation: We report the case of a 69-year-old woman with type 2 diabetes who presented with abnormal movements of the right upper limb. She had no neurological signs other than hemichorea. Her blood glucose level was $349 \mathrm{mg} / \mathrm{dl}$ and her glycosylated haemoglobin level ( $\mathrm{HbA1c}$ ) was $10.5 \%$. Head CT and MRI showed no changes in the basal ganglia or ischaemic lesions. The patient was started on insulin and haloperidol with clinical improvement.

Conclusion: Larger case series are needed to establish better understanding of the physiopathological mechanisms and diagnostic criteria of hyperglycaemic hemichorea. The most important diagnostic criterion is clinical improvement after glycaemic control.

\section{LEARNING POINTS}

- Hyperglycaemia is a rare cause of hemichorea.

- Better understanding of the physiopathology and the establishment of diagnostic criteria are required.

- Correction of the underlying hyperglycaemia will lead to rapid improvement of the movements and is the most important feature for diagnosis.

\section{KEYWORDS}

Hemichorea, diabetes complications, movement disorders

\section{INTRODUCTION}

Chorea is a hyperkinetic movement disorder caused by basal ganglia lesions ${ }^{[1]}$. Hyperglycaemic states are a rare cause and frequently misdiagnosed ${ }^{[1]}$.

Hyperglycaemic hemichorea is characterized by clinical, imaging and treatment criteria. Patients present with involuntary randomappearing, irregular, non-rhythmic lateralized movements on one side of the body, contralateral basal ganglia lesions on head CT scans or MRI, and clinical improvement after glycaemia normalization ${ }^{[2]}$. 
It is also important to carry out a thorough differential diagnosis to rule out ischaemic cerebrovascular, inflammatory, infectious, neurodegenerative and other metabolic diseases ${ }^{[3]}$.

We present the case of a 69-year-old woman with uncontrolled type 2 diabetes mellitus with chorea-like movements. Her symptoms resolved after glycaemic control was established.

\section{CASE DESCRIPTION}

A 69-year-old woman presented with abnormal movements of the right upper limb which had begun 7 days previously. These movements were involuntary, rapid and irregular and did not spread to her torso, other limbs or face. No other conditions such as visual complaints, dizziness, limb weakness, facial deviation, slurred speech, squinting or double vision were present.

The patient had a previous history of hypertension, dyslipidaemia, type 2 diabetes and diabetic retinopathy. She was medicated with candesartan, hydrochlorothiazide, furosemide, simvastatin, metformin, sitagliptin and glimepiride. She did not smoke, drink alcohol or use illicit drugs. She did not have any family history of neurological disease.

On examination she was conscious, her blood pressure was $126 / 61 \mathrm{mmHg}$, and she had no fever or cognitive impairment. She had no other neurological signs.

Her blood glucose level was $349 \mathrm{mg} / \mathrm{dl}$ (Table 1) and her glycosylated haemoglobin level (HbA1c) was $10.5 \%$ with no urinary ketones and with normal renal and liver function. A head CT scan showed an unspecific calcification in the right corona radiata and was negative for acute ischaemia, bleeding or mass in the vicinity of the basal ganglia.

\begin{tabular}{|l|l|l|}
\hline & Values & Normal range \\
\hline Leucocytes & $5.80 \times 10^{9} / \mathrm{l}$ & $4.50-11.50$ \\
\hline Neutrophils & $63.9 \%$ & \\
\hline Platelet count & $289 \times 10^{9} / \mathrm{l}$ & $150-450 \times 10^{9} / \mathrm{l}$ \\
\hline ESR & $21 \mathrm{sec}$ & $0-20$ \\
\hline Urea & $47 \mathrm{mg} / \mathrm{dl}$ & $16-42$ \\
\hline Creatinine & $0.8 \mathrm{mg} / \mathrm{dl}$ & $0.5-1.2$ \\
\hline Sodium & $138 \mathrm{mEg} / \mathrm{l}$ & $136-145$ \\
\hline Potassium & $4.3 \mathrm{mEq} / \mathrm{l}$ & $3.4-4.4$ \\
\hline Glucose & $349 \mathrm{mg} / \mathrm{dl}$ & $74-106$ \\
\hline AST & $31 \mathrm{IU} / \mathrm{l}$ & $3-31$ \\
\hline ALT & $43 \mathrm{IU} / \mathrm{l}$ & $3-31$ \\
\hline CRP & $0.24 \mathrm{mg} / \mathrm{dl}$ & $<0.50$ \\
\hline HbA1c & $10.5 \%$ & $4.0-6.5$ \\
\hline TSH & $1.39 \mathrm{mIU} / \mathrm{l}$ & $4.0-6.5$ \\
\hline T4 & $1.0 \mathrm{ng} / \mathrm{dl}$ & $0.9-1.8$ \\
\hline Cholesterol & $284 \mathrm{mg} / \mathrm{dl}$ & $\leq 200$ \\
\hline LDL & $206 \mathrm{mg} / \mathrm{dl}$ & $<221$ \\
\hline
\end{tabular}

Table 1: Laboratory test
ALT - aspartate aminotransferase; AST - aspartate transaminase;
HbA1c - glycosylated haemoglobin; CRP - C reactive protein;
ESR - erythrocyte sedimentation rate; TSH - thyroid stimulating hormone

The patient was started on valproic acid and clonazepam with no clinical improvement.

She was admitted to our care for observation and treatment with levetiracetam.

An electroencephalogram did not show an epileptogenic pattern. A head MRI revealed no basal ganglia lesions, but did show T1 bilateral hyperdensities in subcortical regions and T2 hypodensity in white matter reported as a calcified lesion. 
Due to the suspicion of hyperglycaemic hemichorea, the patient was treated with NPH insulin ( 4 units in the morning, 6 units at lunch and 10 units at dinner), glargine (22 units in the morning and 8 units at night) and haloperidol $1 \mathrm{mg}$ in the morning and $2 \mathrm{mg}$ at night. Blood glucose levels were maintained at 113-200 mg/dl during hospitalization and showed clinical improvement after 2 days of treatment.

The patient was discharged 14 days later with mild choreiform movements and prescribed a pre-mixed insulin $(75 \%$ NPH and $25 \%$ insulin lispro), 20 units in the morning and 10 units every night, and the same dose of haloperidol. After 2 weeks, at the first follow-up appointment, the patient denied new episodes of chorea, experiencing just the occasional tremor. Five months later, the patient had good insulin therapy adherence, was on a lower dose of haloperidol, and had a HbA1c of $6.5 \%$.

\section{DISCUSSION}

Hyperglycaemic chorea was described for the first time by Bedwell in 1960 . He analysed 53 cases with mean of age 71 years who were predominantly Asian women, suggesting a genetic predisposition ${ }^{[4]}$.

In 2017, the aetiology and pathophysiology of hyperglycaemic chorea are still unknown ${ }^{[3,5]}$. However, it is more frequently seen in patients with longstanding, poorly controlled type 2 diabetes mellitus but has also been reported in type 1 diabetes or as an initial presentation of diabetes $^{[1]}$.

Many mechanisms have been proposed for hyperglycaemic hemichorea. One theory suggests transient ischaemia caused by cerebrovascular insufficiency, petechial haemorrhage and hyperglycaemic hyperosmolarity with reduction in cerebral blood flow, which could explain the hyperintense signal on T1-weighted (T1W) images in MRI in basal ganglia ${ }^{[5]}$. It is also known that both gamma aminobutyric acid (GABA) and acetylcholine are alternative sources of energy in anaerobic pathways, as in hyperglycaemic states. The depletion of GABA causes metabolic acidosis and depression of Kreb's cycle, while a reduction in acetate production causes depletion of acetylcholine ${ }^{[1,5]}$, causing thalamus disinhibition and hyperkinesias ${ }^{[1]}$. Oestrogen-induced alterations in GABA and dopamine receptors in postmenopausal women can be observed, explaining the female predisposition ${ }^{[2]}$.

Diagnosis is based on a triad of clinical, imaging and treatment criteria. Patients have unilateral, involuntary, random appearing movements. A head CT scan usually shows hyperdense unilateral or bilateral lesions of the basal ganglia, while the most common features seen on head MRI are hyperintense signals in the contralateral putamen on T1W images without surrounding oedema or mass effect ${ }^{[5]}$. Abnormal movements are reversible after blood glucose normalization ${ }^{[3]}$, while slower improvement can be associated with typical or atypical antipsychotics, dopamine-depleting agents, benzodiazepines and anticonvulsants ${ }^{[5]}$.

The differential diagnosis includes ischaemic/haemorrhagic stroke, encephalitis, vasculitis, systemic and central nervous system lupus, cerebral toxoplasmosis associated with AIDS, mass lesions, multiple sclerosis, Wilson's disease, thyrotoxicosis and the side effects of drugs such as anticonvulsants, levodopa, oral contraceptives and neuroleptics ${ }^{[2,3]}$.

Prognosis is good, with remission of the symptoms in a few days, although abnormalities persisting for as long as 6 years have been reported ${ }^{[5]}$. $\mathrm{CT}$ and $\mathrm{MRI}$ abnormalities usually return to normal within weeks to months with or after the disappearance of abnormal movements ${ }^{[5]}$.

We report a case of hyperglycaemic hemichorea in a woman with uncontrolled diabetes with retinopathy and $\mathrm{HbA} 1 \mathrm{c}$ of $10.5 \%$. The patient had some of the clinical and treatment criteria of the diagnostic triad but did not show changes in basal ganglia on head CT scan or MRI. This presentation has been reported before and could be explained by depletion of both GABA and acetylcholine ${ }^{[1,5]}$.

\section{CONCLUSION}

Larger case series are needed to establish better understanding of the physiopathological mechanisms and diagnostic criteria for hyperglycaemic hemichorea. The diagnostic is difficult and a normal head CT or MRI does not rule out this condition. it is important to take a thorough history, rule out other causes of hemichorea and achieve clinical improvement with glycaemic control.

\section{REFERENCES}

1. Yassin AM, Shroff S, Patel SD, Parker AM, Berman MA, Jackson GR, et al. Hemichorea in a patient with diabetic ketoacidosis. J Neurol Sci 2014;342:189-191.

2. Bizet J, Cooper CJ, Quansah R, Rodriguez E, Teleb M, Hernandez GT. Chorea, hyperglycemia, basal ganglia syndrome (C-H-BG) in an uncontrolled diabetic patient with normal glucose levels on presentation. Am J Case Rep 2014;15:143-146.

3. Xin Qi, Ya-yun Yan, Yao Gao, Zhao-shi Zheng, Ying Chang. Hemichorea associated with non-ketotic hyperglycemia: a case report. Diabetes Res Clin Pract 2012;95:e1-e3.

4. Bhagwat NM, Joshi AS, Rao G, Varthakavi PK. Uncontrolled hyperglycaemia: a reversible cause of hemichorea-hemiballismus. BMJ Case Rep 2013; doi: 10.1136/bcr-2013010229.

5. Massaro F, Palumbo P, Falcini M, Zanfranceschi G, Pratesi A. Generalized chorea-ballism in acute non ketotic hyperglycemia: findings from diffusion-weighted magnetic resonance imaging. Parkinsonism Relat Disord 2012;18:998-999. 